\title{
Clinical characteristics of COVID-19 patients admitted at the Korle-Bu Teaching Hospital, Accra, Ghana
}
Patrick Adjei $^{1,2}$, Jane Afriyie-Mensah ${ }^{1,2}$, Vincent J. Ganu ${ }^{2}$, Peter Puplampu ${ }^{1,2}$, Bismark Opoku-As- are $^{2}$, Klenam Dzefi-Tettey ${ }^{3}$, Maame-Boatemaa Amissah-Arthur ${ }^{1,2}$, Kenneth Tachi ${ }^{1,2}$, Vincent Boima $^{1,2}$, Dzifa Dey ${ }^{1,2}$ Joseph Akamah ${ }^{1,2}$, Albert Akpalu, ${ }^{1,2}$, Josephine Akpalu, ${ }^{1,2}$, Phillip Amoo ${ }^{4}$, Elom Otchi ${ }^{4}$, Kissinger Marfoh ${ }^{4}$ and Adwoa Agyei-Nkansah ${ }^{1,2}$

Ghana Med J 2020; 54(4) supplement: 33-38 doi: http://dx.doi.org/10.4314/gmj.v54i4s.6

\author{
${ }^{1}$ Department of Medicine and Therapeutics, University of Ghana Medical School, University of Ghana, Accra, \\ Ghana \\ ${ }^{2}$ Department of Medicine, Korle Bu Teaching Hospital, Accra, Ghana \\ ${ }^{3}$ Department of Radiology, Korle Bu Teaching Hospital, Accra, Ghana \\ ${ }^{4}$ Public Health Unit, Korle Bu Teaching Hospital, Accra, Ghana
}

Corresponding author: Dr Patrick Adjei

E-mail: padjei@ug.edu.gh

\author{
Conflict of interest: None declared
}

\section{SUMMARY}

The study examined the clinical characteristics and outcomes of 2019 novel coronavirus disease (COVID-19) infections among hospitalized patients.

Design: Study design was a retrospective single-center review of hospital data.

Setting: The study was conducted at the COVID-19 Treatment Center of the Department of Medicine and Therapeutics of the Korle-Bu Teaching hospital in Accra, Ghana.

Participants and study tools: A total of fifty patients with laboratory (rRT-PCR) confirmed COVID-19 infection were involved in the study. A chart review of the medical records of the patients was conducted and the data obtained was documented using a data extraction form.

Results: The median age was 53 years and most $(36 \%(18 / 50))$ of the patients were at least 60 years of age. Eighty percent (40/50) of the patients were symptomatic, with cough and difficulty in breathing being the commonest presenting symptoms. The mean duration of hospitalization was $12.3 \pm 7.3$ days. Hypertension and Diabetes Mellitus were the commonest co-morbidities occurring in 52\% (26/50) and 42\% (21/50) of patients respectively. Fifty percent of patients developed COVID-19 pneumonia as a complication. The mortality rate was $12 \%(6 / 50)$.

Conclusion: In this study, SARS-CoV2 infection affected older adults with hypertension and diabetes mellitus being the common comorbidities. Patients with these comorbid conditions should be counselled by their clinicians to strictly observe the COVID-19 prevention protocols to reduce their risk of acquiring the infection. There is a need to pay critical and prompt attention to the management of patients with COVID-19 pneumonia particularly among people with diabetes to improve outcomes.

Keywords: COVID-19, hospitalized, symptoms, complications, screening, pneumonia Funding: None declared

\section{INTRODUCTION}

The 2019 novel coronavirus disease (COVID-19) is a communicable respiratory disease caused by a novel strain of coronavirus suspected to be of zoonotic origin that causes illness in human beings. ${ }^{1,2}$ The novel coronavirus infection was first reported by China on $29^{\text {th }}$ December 2019 and rapidly evolved into a pandemic affecting many countries. The disease is spread from person to person through infected droplets from sneezing, coughing or talking.
Transmission can also be through physical contact of hands with infected surfaces and touching of face with contaminated hands. Transmission from asymptomatic contacts has been reported.,

The novel coronavirus pandemic moved swiftly from China and Asia to Europe and the Americas before Africa started experiencing its initial cases. 


\section{Original Article}

The first case of COVID-19 infection in Africa was reported in Egypt ${ }^{5}$ in February 2020 with Nigeria reporting the first COVID-19 case in Sub-Saharan Africa. ${ }^{6}$

As at $19^{\text {th }}$ July 2020 , the total number of confirmed cases worldwide was over 14 million with over 600,000 deaths. ${ }^{7}$ Of these, Africa had over 700,000 cases with almost 15,000 deaths. South Africa had the highest of number of cases $(350,879)$ and deaths $(4948)$ in Africa with Ghana being the third country with the highest number of confirmed cases (27060) with 145 deaths. ${ }^{7}$

Although countries in Africa including Ghana are publicly reporting overall COVID-19 infection numbers and deaths regularly, there is little or no information on the clinical information of patients infected with COVID-19 infection in Ghana. Data on the epidemiological, clinical and laboratory characteristics of patients with COVID-19 infection have been reported in studies conducted in Asia (mainly China) with few from Europe. , $^{8,18-20,10-17}$

There is little of such information from countries in Africa including Ghana since the first recorded cases in Africa. Analysis of such data is required to inform health managers and service providers of early findings amongst our patients, which is needed for targeted management, prevention and health education approaches.

This study sought to determine the clinical characteristics, baseline co-morbidities and clinical outcomes of COVID-19 infected patients in Ghana.

\section{METHODS}

A retrospective single-centre review of hospital data was conducted among patients with laboratory confirmed diagnosis of SARS-CoV-2 infection and admitted to the COVID Treatment Center at the Medical Department of the Korle-Bu Teaching hospital from $16^{\text {th }}$ April to $30^{\text {th }}$ June 2020. The laboratory confirmation of the SARS$\mathrm{CoV}-2$ infection amongst these patients were done using 2019-nCoV rRT-PCR at the Noguchi Memorial Institute for Medical Research (NMIMR) in Accra Ghana. Samples were obtained using nasopharyngeal swabs according to the recommended protocol by the Ghana National Covid-19 Guidelines. ${ }^{21}$.

Any patient with confirmed SARS-CoV-2 infection presenting with no clinical symptoms and signs from date of admission to discharge from hospital was classified as an asymptomatic patient. Patients with confirmed SARS$\mathrm{CoV}-2$ infection with any clinical symptom or sign from date of admission to discharge from hospital was classified as a symptomatic patient.
A chart review of the medical records of the patients was conducted. Data was obtained from the medical records using a data extraction form. Data extracted from the medical files of patients included patients' socio-demographic characteristics and clinical characteristics.

The sociodemographic characteristics extracted included age, sex and occupation. Data on clinical characteristics extracted included clinical history, co-morbidities, duration of admission, complications developed during admission and clinical outcomes. Complications were described as Covid Pneumonia on Chest CT scan, Acute Kidney Injury (AKI), Deep Vein Thrombosis (DVT), Pleural Effusion whilst clinical outcomes were categorized into discharge from hospital and death from SARSCOV2 or its complications. The data extracted were reviewed by 2 independent research investigators as part of data validation and quality checks.

The study was conducted in accordance with the principles of Helsinki Declaration on Ethical Principles in human subject research. The Scientific and Technical Committee (STC) and the Institutional Review Board (IRB) of the Korle-Bu Teaching hospital approved this study with study ID KBTH STC/IRB 00095/2020.

\section{Statistical analysis}

The data was entered into an excel sheet and exported into STATA version 13 for analysis. All categorical data were analyzed and presented as frequencies and percentages. The categorical data were compared using the chi square test or, if the data had less than ten expected frequencies, the Fischer's exact test was used. The chi square test was used to compare clinical symptoms to clinical outcomes and also to compare the effects of having a co-morbidity and the clinical outcome. Continuous variables were analyzed and presented as means with standard deviations, or median with quartile ranges. Significance level for all statistical analysis was set at a pvalue less than 0.05 .

\section{RESULTS}

A total of 50 patients diagnosed with COVID-19 were admitted to the hospital of which $50 \%$ were female. The median age was 53 years with an age range of 9-86 years. Majority $(36 \%(18 / 50))$ of the patients were at least 60 years of age (Table 1). More than half $(64 \%(32 / 50))$ of the patients developed complications during admission.

Twenty percent $(10 / 50)$ of patients were asymptomatic. For those who were symptomatic, cough $(57.5 \%$ (23/40)) and difficulty in breathing $(52.5 \%(21 / 40)$, fever $(45.0 \%$, $18 / 40)$ and general malaise (20\%) were the commonest presenting symptoms in the patients. Other complaints (23\%) were headache, chest pain, diarrhea, confusion, chills, anorexia, abdominal pain, sore throat and anosmia. 


\section{Duration of Hospitalization and Co-morbidities}

The mean duration of hospitalization/admission was 12.3 \pm 7.3 days with a range of 1 to 32 days. Twenty four percent $(12 / 50)$ of the patients did not have any comorbidity and hypertension was the commonest co-morbidity occurring in $52 \%(26 / 50)$ of patients (Table 2$)$.

Table 1 Demographic and clinical characteristics of hospitalized patients with COVID-19 at the Korle-Bu Teaching hospital, April - June 2020

\begin{tabular}{|l|l|}
\hline \multicolumn{1}{|c|}{ Item } & Frequency (\%) \\
\hline Age & \\
\hline Median (LQ, UQ) & $53(36,65)$ \\
\hline$<\mathbf{2 5}$ & $3(6 \%)$ \\
\hline $\mathbf{2 5 - 3 4}$ & $9(18 \%)$ \\
\hline $\mathbf{3 5 - 4 4}$ & $6(12 \%)$ \\
\hline $\mathbf{4 5 - 5 9}$ & $14(28 \%)$ \\
\hline $\mathbf{2 6 0}$ & $18(36 \%)$ \\
\hline Sex & \\
\hline Female & $25(50 \%)$ \\
\hline Male & $25(50 \%)$ \\
\hline Symptoms & \\
\hline Asymptomatic & $10(20 \%)$ \\
\hline Symptomatic & $40(80 \%)$ \\
\hline Number of Symptoms & \\
\hline 0 & $10(20 \%)$ \\
\hline 1 & $11(22 \%)$ \\
\hline$\geq 2$ & $29(58 \%)$ \\
\hline Complication & \\
\hline No & $18(36 \%)$ \\
\hline Yes & $32(64 \%)$ \\
\hline Number of Complications & \\
\hline 1 & $30(93.7 \%)$ \\
\hline 2 & $2(6.3 \%)$ \\
\hline $\begin{array}{l}\text { Types of complications } * \text { mul- } \\
\text { tiple responses }\end{array}$ & \\
\hline COVID-19 Pneumonia & $27(84.4 \%)$ \\
\hline Acute Kidney Injury (AKI) & $5(15.6 \%)$ \\
\hline Deep Vein Thrombosis (DVT) & $1(3.1 \%)$ \\
\hline Pleural Effusion & $1(3.1 \%)$ \\
\hline Co-morbidity & $12(24 \%)$ \\
\hline No & $38(76 \%)$ \\
\hline Yes & \\
\hline Outcome & $44(88 \%)$ \\
\hline Discharged & $6(12 \%)$ \\
\hline Died & \\
\hline Q & \\
\hline
\end{tabular}

$\mathrm{UQ}=$ upper quadrant, $\mathrm{LQ}=$ lower quadrant, $\mathrm{AKI}=$ acute kidney injury, DVT=deep vein thrombosis

Table 2: Co-morbidities among study participants (multiple responses)

\begin{tabular}{ll}
\hline Comorbid condition & N (\%) \\
\hline None & $12(24 \%)$ \\
\hline Hypertension & $26(52 \%)$ \\
\hline Diabetes Mellitus & $21(42 \%)$ \\
\hline Kidney Disease & $8(16 \%)$ \\
\hline CVD & $7(14 \%)$ \\
\hline Asthma & $3(6 \%)$ \\
\hline Retroviral Infection & $3(6 \%)$ \\
\hline Others & $5(10 \%)$ \\
\hline
\end{tabular}

The other conditions comprised allergic rhinitis, post thyroidectomy left vocal cord paralysis, bleeding hemorrhoids, prostate cancer and deep vein thrombosis.

\section{Comorbid Condition, Symptoms at admission and the Development of Complications}

Patients who had diabetes mellitus and hypertension showed statistically significant associations to developing complications $(\mathrm{p}=0.001)$ and $(\mathrm{p}=0.01)$ respectively. Patients who showed symptoms at the time of admission were also more likely to have complications $(\mathrm{p}=0.001)$ (Table 3).

Table 3 Presence of Co-morbid Condition, symptoms at admission and the development of complications among study participants

\begin{tabular}{|c|c|c|c|}
\hline \multicolumn{4}{|c|}{ Complication } \\
\hline CO-MORBIDITY & No $(\%)$ & Yes $(\%)$ & P-value \\
\hline \multicolumn{4}{|l|}{ Diabetes Mellitus } \\
\hline No & $16(55.17)$ & $13(44.83)$ & \\
\hline Yes & $2(9.52)$ & $19(90.48)$ & 0.001 \\
\hline \multicolumn{4}{|l|}{ Hypertension } \\
\hline No & $13(54.17)$ & $11(45.83)$ & \\
\hline Yes & $5(19.23)$ & $21(80.77)$ & 0.01 \\
\hline \multicolumn{4}{|l|}{ Asthma } \\
\hline No & $16(34.04)$ & $31(65.96)$ & \\
\hline Yes & $2(66.67)$ & $1(33.33)$ & 0.291 \\
\hline \multicolumn{4}{|c|}{ Cardiovascular disease } \\
\hline No & $17(39.53)$ & $26(60.47)$ & \\
\hline Yes & $1(14.29)$ & $6(85.71)$ & 0.398 \\
\hline \multicolumn{4}{|l|}{ Kidney disease } \\
\hline No & $16(38.1)$ & $26(61.9)$ & \\
\hline Yes & $2(25)$ & $6(75)$ & 0.694 \\
\hline \multicolumn{4}{|l|}{ HIV Infection } \\
\hline No & $18(38.3)$ & $29(61.7)$ & \\
\hline Yes & $0(0)$ & $3(100)$ & 0.544 \\
\hline \multicolumn{4}{|l|}{ SYMPTOMS } \\
\hline Asymptomatic & $9(100)$ & $0(0)$ & \\
\hline Symptomatic & $9(21.95)$ & $32(78.05)$ & $<0.001$ \\
\hline \multicolumn{4}{|c|}{ Number of Symptoms } \\
\hline $\mathbf{0}$ & $9(100)$ & $0(0)$ & \\
\hline 1 & $5(38.46)$ & $8(61.54)$ & \\
\hline$\geq 2$ & $4(14.29)$ & $24(85.71)$ & $<0.001$ \\
\hline
\end{tabular}

\section{Clinical Outcomes}

The mortality rate among the number of patients admitted and managed for COVID-19 was $12 \%(6 / 50)$. There was no significant association between age and clinical outcomes $(p=0.95)$. No significant association between sex and clinical outcomes $(p=0.19)$ was observed (Table 3$)$. All 6 patients who died had at least one co-morbidity. Five of them had both hypertension and diabetes mellitus whilst one had HIV infection. There was no statistically significant association between having any co-morbidity and the clinical outcome (See Table 4). 
Table 4 COVID-19 complications, Comorbid Condition, Age, Gender and the clinical outcomes among study participants

\begin{tabular}{|c|c|c|c|}
\hline & \multicolumn{3}{|l|}{ Outcome } \\
\hline & Died & $\begin{array}{l}\text { Dis- } \\
\text { charged }\end{array}$ & $\begin{array}{l}P- \\
\text { value }\end{array}$ \\
\hline Age & & & 0.953 \\
\hline$<25$ & $0(0)$ & $3(100)$ & \\
\hline $25-34$ & $1(11.11)$ & $8(88.89)$ & \\
\hline $35-44$ & $0(0)$ & $6(100)$ & \\
\hline 45-59 & $2(14.29)$ & $12(85.71)$ & \\
\hline$\geq 60$ & $3(16.67)$ & $15(83.33)$ & \\
\hline Sex & & & 0.189 \\
\hline Female & $5(20)$ & $20(80)$ & \\
\hline Male & $1(4)$ & $24(96)$ & \\
\hline \multicolumn{4}{|c|}{ Co-Morbidity } \\
\hline \multicolumn{4}{|c|}{ Diabetes Mellitus } \\
\hline No & $1(3.45)$ & $28(96.55)$ & \\
\hline Yes & $5(23.81)$ & $16(76.19)$ & 0.07 \\
\hline \multicolumn{4}{|c|}{ Hypertension } \\
\hline No & $1(4.17)$ & $23(95.83)$ & \\
\hline Yes & $5(19.23)$ & $21(80.77)$ & 0.192 \\
\hline \multicolumn{4}{|l|}{ Asthma } \\
\hline No & $6(12.77)$ & $41(87.23)$ & \\
\hline Yes & $0(0)$ & $3(100)$ & 1 \\
\hline \multicolumn{4}{|c|}{ *Cardiovascular disease } \\
\hline No & $5(11.63)$ & $38(88.37)$ & \\
\hline Yes & $1(14.29)$ & $6(85.71)$ & 1 \\
\hline \multicolumn{4}{|c|}{ Chronic Kidney Disease } \\
\hline No & $5(11.9)$ & $37(88.1)$ & \\
\hline Yes & $1(12.5)$ & $7(87.5)$ & 1 \\
\hline \multicolumn{4}{|c|}{ HIV Infection } \\
\hline No & $5(10.64)$ & $42(89.36)$ & \\
\hline Yes & $1(33.33)$ & $2(66.67)$ & 0.324 \\
\hline \multicolumn{4}{|c|}{ Symptoms } \\
\hline \multicolumn{4}{|l|}{ Cough } \\
\hline No & $4(14.81)$ & $23(85.19)$ & \\
\hline Yes & $2(8.7)$ & $21(91.3)$ & 0.674 \\
\hline \multicolumn{4}{|c|}{ Difficulty in Breathing } \\
\hline No & $2(6.9)$ & $27(93.1)$ & \\
\hline Yes & $4(19.05)$ & $17(80.95)$ & 0.223 \\
\hline \multicolumn{4}{|l|}{ Fever } \\
\hline No & $2(6.25)$ & $30(93.75)$ & \\
\hline Yes & $4(22.22)$ & $14(77.78)$ & 0.171 \\
\hline \multicolumn{4}{|c|}{ General Malaise } \\
\hline No & $4(9.52)$ & $38(90.48)$ & \\
\hline Yes & $2(25)$ & $6(75)$ & 0.242 \\
\hline \multicolumn{4}{|c|}{ Number of Symptoms } \\
\hline $\mathbf{0}$ & $0(0)$ & $9(100)$ & \\
\hline 1 & $0(0)$ & $13(100)$ & \\
\hline$\geq 2$ & $6(21.43)$ & $22(78.57)$ & 0.112 \\
\hline \multicolumn{4}{|c|}{ Complication } \\
\hline No & $0(0)$ & $18(100)$ & \\
\hline Yes & $6(18.75)$ & $26(81.25)$ & 0.071 \\
\hline
\end{tabular}

\section{DISCUSSION}

The study sought to describe the preliminary clinical findings from management of patients with COVID-19 at a tertiary hospital in Ghana. Our early findings showed that majority of our patients were at least 60 years old.
Eighty percent of patients presented with symptoms with cough being the commonest. More than half of the patients had at least 2 symptoms.

Sixty-four percent of the patients developed complications with the commonest complication being Covid pneumonia. Seventy-five percent had co-morbidities with hypertension being the commonest co-morbidity and the mortality rate was $12 \%$.

The median age in our study was similar to findings in other studies including a meta-analysis study in China $12,13,15$ but lower compared to findings from another study in Italy ${ }^{14}$ and higher than a study in Saudi Arabia. ${ }^{11}$ This may be explained by the fact that adults may be more susceptible to SARS-COV2 infection and subsequent hospitalization as against the younger age group. ${ }^{22}$ However, the spike in the younger age group for the female gender may have been introduced by the small sample size with its attendant low power which may have increased the risk of a negative bias. There was equal distribution of infection with regards to gender which was contrary to reports from other studies where males were found to be more affected than females. ${ }^{8-11}$ The commonest symptom in our patients was cough which was corroborated by reports from previous studies. ${ }^{10,11,19}$ Contrary to other studies, fever was the third commonest symptoms amongst our patients. ${ }^{8,9,23}$

Hypertension was the commonest co-morbidity in our patients followed by diabetes mellitus. This is consistent with findings in other studies including one among an African American population where hypertension was reported as the commonest co-morbidity. $8,10,11,15,19,23$ This finding may also be a reflection of the high prevalence of hypertension and diabetes mellitus in the Ghanaian adult population. ${ }^{24,25}$ Similar to the study by Koh et al, Diabetes mellitus (DM) was the second co-morbidity in our study. Chronic Kidney disease was the third commonest comorbid condition, in contrast to findings by Koh et al who reported cardiovascular disease as the third commonest comorbid condition. ${ }^{13}$ The high prevalence of hypertension and diabetes mellitus in Ghana, the negative effects of these two conditions in the development of chronic kidney disease, as well as, the inadequate resources in managing end stage renal disease leads to an apparent high prevalence of $\mathrm{CKD}^{26,27}$ and may account for the observations in the study. Acute kidney injury has been reported in some studies ${ }^{13,19,20}$ as a complication of the novel coronavirus infection and similar findings were observed in this study. 
Approximately $84 \%$ of the patients in our study developed COVID-19 pneumonia as a complication similar to findings from another study in hospitalized African American population. ${ }^{19}$ Other studies in non-African population also reported interstitial pneumonia as the commonest complication among patients with COVID19. ${ }^{9,14,23}$

There was no significant difference in the distribution of complications between males and females in this study. Development of a complication was found to be closely linked to diabetes mellitus, hypertension and being symptomatic with more than two symptoms at the time of admission. This may be due to the decreased efficiency of both humoral and cellular immunity in diabetes mellitus $^{28}$. Also, elevated levels of cytokines, visceral obesity and state of chronic inflammation in diabetes mellitus may also play a role in the development of complication. ${ }^{29,30}$

The mortality rate in this study was $12 \%$ with half of them being at least 60 years of age. There was no significant association between the clinical outcome and demographic and clinical characteristics. However, having diabetes mellitus and complications was associated with increasing mortality even though this did not have any statistical significance. In the setting of a low powered study such as the present study, increasing the power may support the risk of poor outcomes with the presence of comorbid conditions particularly diabetes mellitus. It is plausible that as diabetes mellitus affects both humoral and T-cell mediated immunity and is a hyper-coagulable state $^{29,30}$, the presence of diabetes mellitus may carry a higher risk of poor outcomes in COVID-19 disease. In addition, T-cell mediated immunity that is typically compromised in HIV infection may have accounted for the lower incidence of poor outcomes in this group compared to other co-morbidities despite the high incidence of opportunistic infections and high mortality of HIV infections.

The limitation of this study is the small sample size. The sample size is small as this study sought to provide preliminary findings on patients managed in our setting. The small sample size is likely to reduce the power of the study and lead to higher variability.

\section{CONCLUSION}

In this study, SARS-CoV2 infection affected older adults with hypertension and diabetes mellitus being the common comorbidities. Patients with these comorbid conditions should be counselled by their clinicians to strictly observe the COVID-19 prevention protocols to reduce their risk of acquiring the infection. There is a need to pay critical and prompt attention to the management of patients with COVID-19 pneumonia particularly among people with diabetes to improve outcomes.

\section{ACKNOWLEDGEMENT}

We acknowledge all Medical Staff of the Department of Medicine, Korle Bu Teaching Hospital

\section{REFERENCES}

1. Zhu N, Zhang D, Wang W, et al. A novel coronavirus from patients with pneumonia in China, 2019. N Engl J Med. 2020;382(8):727-733.

2. Wu Y-C, Chen C-S, Chan Y-J. Overview of The 2019 Novel Coronavirus (2019-nCoV): The Pathogen of Severe Specific Contagious Pneumonia (SSCP). $J$ Chin Med Assoc. 2020;83(3):217-220.

3. Rothe C, Schunk M, Sothmann P, et al. Transmission of 2019-NCOV infection from an asymptomatic contact in Germany. N Engl J Med. 2020;382(10):970971. doi:10.1056/NEJMc2001468

4. Bai Y, Yao L, Wei T, et al. Presumed Asymptomatic Carrier Transmission of COVID-19. JAMA 2020;323(14). doi:10.1001/jama.2020.2565

5. A second COVID-19 case is confirmed in Africa | WHO | Regional Office for Africa. Published 2020. Accessed April 25, 2020. https://www.afro.who.int/news/second-covid-19case-confirmed-africa

6. Coronavirus: Nigeria confirms first case in sub-Saharan Africa - BBC News. 2020. Accessed April 25, $2020 . \quad$ https://www.bbc.com/news/world-africa51671834

7. Worldometer. Coronavirus Cases. Worldometer. doi:10.1101/2020.01.23.20018549V2

8. Zhou F, Yu T, Du R, et al. Clinical course and risk factors for mortality of adult inpatients with COVID19 in Wuhan, China: a retrospective cohort study. Lancet. $\quad 2020 ; 395(10229): 1054-1062$. doi:10.1016/S0140-6736(20)30566-3

9. Guan W, Ni Z, Hu Y, et al. Clinical Characteristics of Coronavirus Disease 2019 in China. N Engl J Med. 2020;382(18):1708-1720.

doi:10.1056/NEJMoa2002032

10. Goyal P, Choi JJ, Pinheiro LC, et al. Clinical Characteristics of Covid-19 in New York City. $N$ Engl $J$ Med. 2020;382(24):2372-2374. doi:10.1056/NEJMc2010419

11. Alsofayan YM, Althunayyan SM, Khan AA, Hakawi AM, Assiri AM. Clinical characteristics of COVID-19 in Saudi Arabia: A national retrospective study. J Infect Public Health. 2020;13(7):920-925. doi:10.1016/j.jiph.2020.05.026

12. Pan L, Mu M, Yang P, et al. Clinical Characteristics of COVID-19 Patients With Digestive Symptoms in 
Hubei, China. Am J Gastroenterol. 2020;115(5):766773. doi:10.14309/ajg.0000000000000620

13. Koh J, Shah SU, Chua PEY, Gui H, Pang J. Epidemiological and Clinical Characteristics of Cases During the Early Phase of COVID-19 Pandemic: A Systematic Review and Meta-Analysis. Front Med. 2020;7:295. doi:10.3389/fmed.2020.00295

14. Colaneri M, Sacchi P, Zuccaro V, et al. Clinical characteristics of coronavirus disease (COVID-19) early findings from a teaching hospital in Pavia, North Italy, 21 to 28 February 2020. Eurosurveillance. 2020;25(16). doi:10.2807/15607917.ES.2020.25.16.2000460

15. Cao Z, Li T, Liang L, et al. Clinical characteristics of Coronavirus Disease 2019 patients in Beijing, China. Feng Y-M, ed. PLoS One. 2020;15(6):e0234764.doi:10.1371/journal.pone. 0234764

16. Huang C, Wang Y, Li X, et al. Clinical features of patients infected with 2019 novel coronavirus in $\mathrm{Wu}-$ han, China. Lancet. 2020;395(10223):497-506. doi:10.1016/S0140-6736(20)30183-5

17. Chen N, Zhou M, Dong X, et al. Epidemiological and clinical characteristics of 99 cases of 2019 novel coronavirus pneumonia in Wuhan, China: a descriptive study. Lancet. 2020;395(10223):507-513. doi:10.1016/S0140-6736(20)30211-7

18. Wang D, Hu B, Hu C, et al. Clinical Characteristics of 138 Hospitalized Patients with 2019 Novel Coronavirus-Infected Pneumonia in Wuhan, China. JAMA - J Am Med Assoc. 2020;323(11):1061-1069. doi:10.1001/jama.2020.1585

19. Suleyman G, Fadel RA, Malette KM, et al. Clinical Characteristics and Morbidity Associated With Coronavirus Disease 2019 in a Series of Patients in Metropolitan Detroit. JAMA Netw open. 2020;3(6):e2012270. doi:10.1001/jamanetworkopen. 2020.12270

20. Richardson S, Hirsch JS, Narasimhan M, et al. Presenting Characteristics, Comorbidities, and Outcomes Among 5700 Patients Hospitalized With COVID-19 in the New York City Area. JAMA. 2020;323(20):2052.

21. Republic of Ghana Ministry of Health Provisional Standard Treatment Guidelines for Novel
Coronavirus Infection COVID-19 Guidelines for Ghana.; 2020. www.ghndp.org

22. Dhochak N, Singhal T, Kabra SK, Lodha R. Pathophysiology of COVID-19: Why Children Fare Better than Adults? Indian J Pediatr. 2020;87(7):537-546.

23. Guo T, Shen Q, Guo W, et al. Clinical Characteristics of Elderly Patients with COVID-19 in Hunan Province, China: A Multicenter, Retrospective Study. Gerontology. Published online May 29, 2020:1-9.

24. Sanuade OA, Boatemaa S, Kushitor MK. Hypertension prevalence, awareness, treatment and control in Ghanaian population: Evidence from the Ghana demographic and health survey. Tayo BO, ed. PLoS One. 2018;13(11):e205985.

25. Asamoah-Boaheng M, Sarfo-Kantanka O, Tuffour AB, Eghan B MJ. Prevalence and risk factors for diabetes mellitus among adults in Ghana: a systematic review and meta-analysis. Int Health. 2019;11(2):8392.

26. Tannor EK, Sarfo FS, Mobula LM, Sarfo-Kantanka O, Adu-Gyamfi R, Plange-Rhule J. Prevalence and predictors of chronic kidney disease among Ghanaian patients with hypertension and diabetes mellitus: A multicenter cross-sectional study. J Clin Hypertens. 2019;21(10):1542-1550. doi:10.1111/jch.13672

27. Boateng EA, East L, Evans C. Decision-making experiences of patients with end-stage kidney disease (ESKD) regarding treatment in Ghana: A qualitative study 11 Medical and Health Sciences 1103 Clinical Sciences 11 Medical and Health Sciences 1117 Public Health and Health Services. BMC Nephrol. 2018;19(1):371.

28. Alves C, Casqueiro J, Casqueiro J. Infections in patients with diabetes mellitus: A review of pathogenesis. Indian J Endocrinol Metab. 2012;16(7):27.

29. Xiao J, Li J, Cai L, Chakrabarti S, Li X. Cytokines and Diabetes Research. J Diabetes Res. 2014;2014. doi:10.1155/2014/920613

30. Randeria SN, Thomson GJA, Nell TA, Roberts T, Pretorius E. Inflammatory cytokines in type 2 diabetes mellitus as facilitators of hypercoagulation and abnormal clot formation. Cardiovasc Diabetol. 2019;18(1):72. 